\title{
OSCILLATORY PROPERTIES OF THE SOLUTIONS OF FIRST ORDER LINEAR NEUTRAL DIFFERENTIAL EQUATIONS WITH "MAXIMA"
}

In this paper we consider a neutral differential equation with "maxima" of the form

$[x(t)+p(t) x(\sigma(t))]+q(t) \max _{[t-\alpha, t]} x(s)=0$

We obtained sufficient conditions for oscillation of all the solutions when $t \rightarrow \infty$.

Keywords: Neutral equation, delayed argument, oscillation.

\section{Introduction}

We consider a first order linear neutral functional differential equation of the form

$$
[x(t)+p(t) x(\sigma(t))]+q(t) \max _{[t-\alpha, t]} x(s)=0, t \geq t_{0}
$$

Let the following conditions regarding functions $p, \sigma, q$ be always assumed to hold:

$$
\begin{aligned}
& t \rightarrow \infty \\
& p:\left[t_{0}, \infty\right) \rightarrow R \text { is continuous } \\
& \sigma:\left[t_{0}, \infty\right) \rightarrow R \text { is continuous and stricly increasing, } \\
& \lim _{t \rightarrow \infty} \sigma(t)=\infty, \alpha \in R, \alpha>0 \\
& q:\left[t_{0}, \infty\right) \rightarrow(0, \infty) \text { is continuous, } \\
& q(t) \neq 0, \int_{0}^{\infty} q(t) d t=0
\end{aligned}
$$

Differential equations with maxima are special type of differential equations that contain the maximum. Though differential equations with maxima are often met in applications, the qualitative theory of these equations is relatively little developed. The existence of periodic solutions of these equations is considered in [1]. Our aim is to obtain new sufficient conditions for the oscillation of all the solutions of equation (1). By the solution of equation (1) we mean a continuous function $x:\left[t_{x}, \infty\right) \rightarrow R$ such that $x(t)+$ $+p(t) x(\sigma(t))$ is continuously differentiable and $x(t)$ satisfies equation (1) for all sufficiently large $t \geq t_{x}$. The vanishing solutions of all large $t$ will be excluded from our consideration. The solution of (1) is called oscillatory if it has arbitrarily large zeros in $\left[t_{x}, \infty\right)$ and it is called nonoscillatory otherwise. The nonoscillating solutions of (1) are characterized as being eventually positive or eventually negative. Let us define the function

$$
z(t)=x(t)+p(t) x(t-\tau)
$$

where $t-\tau=\sigma(t)$. Then, from equation (1) we get

$$
z^{\prime}(t)+q(t) \max _{[t-\alpha, t]} x(s)=0
$$

The following lemmas will be useful in the proof of the main results. Lemmas can be found in [2,3].

\section{Lemma 1.1 We consider equation}

$$
x^{\prime}(t)=p(t) x(t-\tau)=0, t \geq t_{0}
$$

In addition to (2) let us suppose that $p(t)>0, \tau>0,(\sigma(t)<t)$ and

$\lim _{t \rightarrow \infty} \int_{t-\tau}^{t} p(r) d r>\frac{1}{e}$.

Then the following statements are true: the inequality a)

$x^{\prime}(t)+p(t) x(t-\tau) \leq 0$

\footnotetext{
* Zuzana Malacka

Department of Mathematics, Faculty of Humanities, University of Zilina, Slovakia, E-mail: zuzana.malacka@fhv.uniza.sk
} 
has no eventually positive solution,

b)

$x^{\prime}(t)+p(t) x(t-\tau) \geq 0$

has no eventually negative solution, or inequality

c)

$x^{\prime}(t)-p(t) x(t-\tau) \geq 0$

has no eventually positive solution,

d)

$x^{\prime}(t)-p(t) x(t-\tau) \leq 0$

has no eventually negative solution, and

e)

the following equation

$x^{\prime}(t)-p(t) x(t-\tau)=0$

has only oscillatory solutions.

Lemma 1.2 Let the conditions (2) - (4) hold. Then the following statements are true:

a)If $p(t) \leq-1$ and

(i) $x(t)$ is an eventually positive solution of (1), then the function $z(t)$ is an eventually decreasing function and $z(t)<0$ eventually,

(ii) $x(t)$ is an eventually negative solution of (1), then the function $z(t)$ is an eventually increasing function and $z(t)>0$ eventually.

b)If $-1 \leq p(t) \leq 0$ and

(i) $x(t)$ is an eventually positive solution of (1), then the function $z(t)$ is an eventually increasing function and $z(t)>0$ eventually,

(ii) $x(t)$ is an eventually negative solution of (1), then the function $z(t)$ is an eventually decreasing function and $z(t)<0$ eventually.

\section{Main results}

Theorem 2.1 Let conditions (2) - (4) hold and $p(t) \equiv 0$. Then all the solutions of equation (1) are oscillatory.

Theorem 2.2 Let conditions (2) - (4) hold, $p(t) \leq-1$ and $\tau>$ a. Next, let the following condition hold

$$
\liminf _{t \rightarrow \infty} \int_{t-\tau+\alpha}^{t} \frac{q(s)}{\max _{u \in[s-\alpha, s]}\{-p(u+\tau)\}} d s>\frac{1}{e} .
$$

Then all the solutions of equation (1) are oscillatory.
Proof. Let us suppose that equation (1) has a nonoscillatory solution $x(t)$. Let $x(t)<0$. From Lemma (1.2) we have that $z(t)>$ $>0$. Then, from (5) we get the inequality

$$
z(t)<p(t) x(t-\tau)
$$

from which we have

$$
x(t)<\frac{z(t+\tau)}{p(t+\tau)}
$$

and, finally

$$
\max _{[t-\alpha, t]} x(s) \leq \max _{[t-\alpha, t]} \frac{z(s+\tau)}{p(s+\tau)} .
$$

Next, from Lemma (1.2) we know that $z(t)$ is an eventually increasing function, then for sufficiently large $t$ we have

$z(t+\tau-\alpha) \leq z(s+\tau), \mathrm{s} \in[t-\alpha, t]$

Then

$$
\max _{[t-\alpha, t]} \frac{z(t+\tau-\alpha)}{p(s+\tau)} \geq \max _{[t-\alpha, t]} \frac{z(s+\tau)}{p(s+\tau)}
$$

and

$$
\max _{[t-\alpha, t]} \frac{-z(t+\tau-\alpha)}{-p(s+\tau)} \geq \max _{[t-\alpha, t]} \frac{z(s+\tau)}{p(s+\tau)}
$$

From the last inequality and (8) we can deduce that

$$
\max _{[t-\alpha, t]} x(s) \leq \frac{-z(t+\tau-\alpha)}{\max _{[t-\alpha, t]}\{-p(s+\tau)\}} .
$$

Then from (6) and from (9) it follows that only the positive function $z(t)$ satisfies the inequality

$$
z^{\prime}(t)-\frac{q(t)}{\max _{[t-\alpha, t]}\{-p(s+\tau)\}} z(t+\tau-\alpha) \geq 0
$$

But from (7) and from Lemma (1.1) it follows that the last inequality (10) has no positive solutions. The contradiction obtained shows that equation (1) has no negative solutions, which is a contradiction.

Let $x(t)>0$ From Lemma (1.2) it follows that $z(t)<0$ As above, we obtain the estimate

$$
\max _{[t-\alpha, t]} x(s) \geq \frac{-z(t+\tau-\alpha)}{\max _{[t-\alpha, t]}\{-p(s+\tau)\}}
$$

Then from (6) and from (11) it follows that only the negative function satisfies the inequality

$$
z^{\prime}(t)-\frac{q(t)}{\max _{[t-\alpha, t]}\{-p(s+\tau)\}} z(t+\tau-\alpha) \leq 0
$$

But from (7) and from Lemma (1.1) it follows that the last inequality (12) has no negative solutions. Hence equation (1) has no positive solutions, which was a contradiction. 
Then all the solutions of equation (1) are oscillatory.

Theorem 2.3 Let conditions (2) - (4) hold, $-1 \leq p(t) \leq 0$ and $a \geq \tau$. Next, let the following condition hold

$$
\liminf _{t \rightarrow \infty} \int_{t-\tau}^{t} q(s) \max _{u \in[s-a, s]}\{-p(u)\} d s>\frac{1}{e}
$$

Then all the solutions of equation (1) are oscillatory.

Proof. Let us suppose that equation (1) has a nonoscillatory solution .

1) Let $x(t)>0$ From Lemma (1.2) we have that $z(t)$ is a decreasing function and $z(t)>0$. Then $z(t)<x(t)$ and

$$
\max _{[t-\alpha, t]} z(s)<\max _{[t-\alpha, t]} x(s) .
$$

With regard to (6) from the last inequality we get

$z^{\prime}(t)+q(t) \max _{[t-\alpha, t]} z(s) \leq 0$.

Since $z(t)$ is a decreasing function, then we obtain

$$
\max _{[t-\alpha, t]} z(s)=z(t-\alpha) .
$$

Therefore, the positive function $z(t)$ satisfies the inequality

$$
z^{\prime}(t)+q(t) z(t-\alpha) \leq 0
$$

In view of

$$
\max _{[t-\alpha, t]}\{-p(u)\} \leq 1
$$

then from (13) and from Lemma (1.1) it follows that inequality (14) has no positive solutions. The contradiction obtained shows that equation (1) has no positive solutions.

2) Let $x(t)<0$ From Lemma (1.2) we have that $z(t)$ is an increasing function and $z(t)<0$. From the definition of $z(t)$ the inequalities follow

$$
\begin{aligned}
& x(t)<-p(t) x(t-\alpha)<-p(t) z(t-\alpha) . \\
& \max _{[t-\alpha, t]} x(s)<\max _{[t-\alpha, t]}\{-p(s) x(s-\alpha)\}<\max _{[t-\alpha, t]}\{-p(s) z(t-\alpha)\} .
\end{aligned}
$$

With regard to (6) from the last inequality we get

$$
z^{\prime}(t)+q(t) \max _{[t-\alpha, t]}\{-p(s) z(t-\alpha)\}>0 .
$$

From (13) and from Lemma (1.1) it follows that inequality (15) has no negative solutions, which was a contradiction.

Then all the solutions of equation (1) are oscillatory.

\section{Acknowledgment}

This work was supported through the project $041 \mathrm{ZU}-4 / 2011$.

\section{References}

[1] RAYBOV, YU. A., MAGOMEDOV, A. R.: Differential Equations with Maxima [in Russian], Preprint No. 75, Bacou, Institut of Physics Acad. Sci. Azerb, SSR, 2002.

[2] BAINOV, D. D., ZAHARIEV, A. I.: Oscillating and Asymptotic Properties of a Class of Functional Differential Equations with Maxima, Czechoslovak Math. J., 34, 1984, 247-251.

[3] GYORI, I., LADAS, G.: Oscillation Theory of Delay Differential Equations, Clarendon Press, Oxford, 1991. 